\title{
Neuron-Astrocyte Signaling in the Development and Plasticity of Neural Circuits
}

\author{
Beth Stevens \\ Department of Neurobiology, Stanford University School of Medicine, Stanford, Calif., USA
}

\section{Key Words}

CNS injury · CNS synaptogenesis - Contact-dependent astrocyte-neuron signaling $\cdot$ Neurodegenerative disease . Neuron-astrocyte signaling $\cdot$ Spine dynamics $\cdot$ Structural plasticity - Synapse development and structural plasticity . Synapse elimination, role of glia $\cdot$ Refinement of neural circuits

\begin{abstract}
Emerging evidence indicates that signaling between perisynaptic astrocytes and neurons at the tripartite synapse plays an important role during the critical period when neural circuits are formed and refined. Cross-talk between astrocytes and neurons during development mediates synaptogenesis, synapse elimination and structural plasticity through a variety of secreted and contact-dependent signals. Recent live imaging studies reveal a dynamic and cooperative interplay between astrocytes and neurons at synapses that is guided by a variety of molecular cues. A unifying theme from these recent findings is that astrocytes can promote the development and plasticity of synaptic circuits. Insight into the molecular mechanisms by which astrocytes regulate the wiring of the brain during development could lead to new therapeutic strategies to promote repair and rewiring of neural circuits in the mature brain following CNS injury and neurodegenerative disease.
\end{abstract}

Copyright $\odot 2008$ S. Karger AG, Basel

\section{Introduction}

It is becoming increasingly clear that glia can no longer be regarded as passive bystanders at the synapse. We now appreciate that glia actively communicate with preand postsynaptic neurons and influence functions that have long been thought to be under neuronal control. In the grey matter, the intricate processes of protoplasmic astrocytes contact and envelope synaptic terminals $[1,2]$ (fig. 1). Active, bidirectional signaling between neurons and astrocytes influences synaptic function and plasticity in the mature brain, and this has been the subject of many recent reviews [3-5]. Emerging evidence also indicates that neuron-astrocyte signaling plays a pivotal role in the developing brain during the dynamic period when neural circuits are being formed and refined. Cross-talk between immature astrocytes and neurons during this critical period influences the formation and elimination of synapses, as well as synaptic morphology and structural plasticity $[6,7]$.

Astrocytes are well equipped and uniquely positioned to engage in a dynamic two-way dialogue with neurons. Recent advances in our ability to visualize astrocytes in vivo have dramatically changed our views of astrocyte morphology and the extent of neuron-glia interactions at synapses $[8,9]$. Three-dimensional reconstructions of dye-filled astrocytes reveal that astrocytes extend thousands of intricate processes that are organized into large, 
non-overlapping anatomical domains. In fact, it has been estimated that a single astrocyte can associate with multiple neurons, and over 100,000 synapses $[2,10]$ (fig. 1). Two photon confocal time-lapse imaging studies are revealing a dynamic and coordinated interplay between astrocytic processes and dendritic spines in regions of synaptic activity, suggesting astrocytes, like neurons, can rapidly respond to environmental cues to influence synaptic structure and function. Although incapable of firing action potentials, astrocytes secrete a wide array of neuroactive 'gliotransmitters' and trophic factors, and they express many of the same channels, receptors, and cell surface molecules that neurons do $[3,5,11]$. Together these findings place astrocytes in a central position to actively signal with neurons to coordinate developing neural circuits.

One of the first clues that immature astrocytes might play an instructive role in developmental plasticity of neural circuits came from a study in 1989 by Muller and Best [12] that found that injection of immature astrocytes into the adult visual cortex of cats reopened the window of ocular dominance (OD) plasticity, a well-characterized form of experience-dependent synaptic remodeling. If vision is restricted to only one eye early in development, the territories (OD columns) innervated by the deprived eye shrink dramatically, whereas those innervated by the non-deprived eye expand. After this 'critical period', monocular deprivation is normally unable to induce OD plasticity, but transplantation of astrocytes derived from newborn kittens was sufficient to reintroduce OD plasticity in adult animals [12]. This was a surprising finding at the time, as astrocytes were generally thought to play a passive, supportive role at the synapse.

Muller [13] proposed that 'structural changes in neuronal connectivity can be influenced or mediated by glial cells via release of growth or growth permissive factors on neuronal activation, and by active displacement and subsequent elimination of axonal boutons'. Muller and Best [12] further hypothesized that immature astrocytes are a requisite for visual cortical plasticity, and that the end of the critical period is linked to the maturation of astrocytes. Consistent with this idea, chondroitin sulfate proteoglycans (CSPGs), extracellular matrix proteins produced by mature astrocytes, are inhibitory for experiencedependent plasticity. Enzymatic degradation of CSPGs reactivates OD plasticity in the adult visual cortex [14].

OD plasticity is likely mediated by both the elimination of synaptic inputs from the deprived eye, and the expansion and formation of new synapses by inputs from the open eye. While the specific astrocyte-derived signals that mediate the reinduction of OD plasticity are not yet
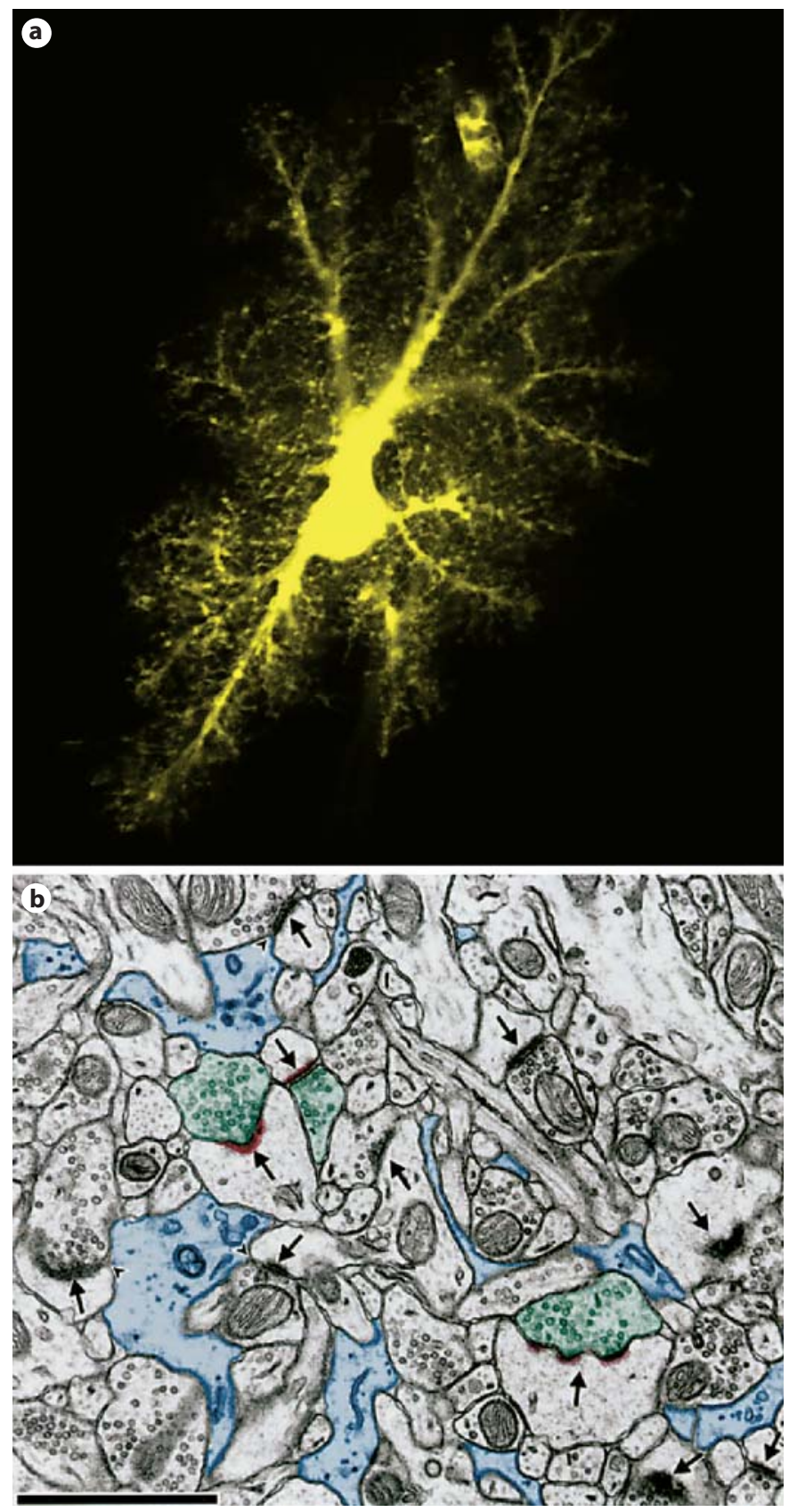

Fig. 1. Protoplasmic astrocytes are intimately associated with synapses. a Protoplasmic astrocyte of CA1 region of rat hippocampus filled with Lucifier Yellow dye revealed thousands of dense spongiform processes that ensheath synapses (provided by Eric Bushong [2]). b Electron micrograph of the 'tripartite synapse' in the rodent brain, showing several synapses (arrows) ensheathed by astrocytic processes (blue). From Kristen Harris http://synapse-web.org/anatomy/astrocyte/Astrocyte.stm. 
clear, this important study provided the first evidence that immature astrocytes play an active role in developmental plasticity in vivo. More recently, in vitro studies have identified several secreted and contact-dependent astrocyte-derived signals that mediate synapse formation, elimination, and structural plasticity.

This review highlights recent examples of neuron-astrocyte synaptic signaling in the developing and plastic brain. Understanding the mechanisms and signaling molecules that control the wiring of the brain during the postnatal period could also lead to new therapeutic strategies to promote repair and rewiring of neural circuits in the mature brain, especially following CNS injury and neurodegenerative disease.

\section{Secreted Astrocyte-Derived Signals Regulate CNS Synaptogenesis}

It has long been thought that the process of synaptogenesis is an intrinsic ability of neurons, but research over the past decade has challenged this traditional view. There is a clear spatiotemporal correlation between the onset of synaptogenesis and the appearance and association of immature astrocytes with neurons at CNS synapses [15], suggesting that astrocytes may provide instructive signals to control the formation and development of synapses. Indeed, recent work from many laboratories indicates that astrocytes have a powerful ability to promote excitatory and inhibitory synaptogenesis.

Evidence that astrocytes secrete synaptogenic factors has emerged from several recent in vitro studies. Purified rodent retinal ganglion cells (RGCs) have proven a particularly useful model system to investigate the role of astrocytes in synapse development, as RGCs can be immunopurified and cultured for several weeks in the complete absence of glia and other cell types [16, 17]. RGC neurons exhibit very little spontaneous synaptic activity unless they are cultured with a feeding layer of astrocytes, or astrocyte-conditioned medium (ACM), as measured by electrophysiology and FM1-43 staining [18]. Consistent with these findings, purified spinal motor neurons are not synaptically active unless exposed to astrocytes [19]. Indeed, immunostaining and electron microscoscopy confirmed that the number of structural synapses that formed between cultured RGCs, or between RGCs and their normal target neurons in the superior colliculus, was significantly increased upon exposure to astrocytes [15].
What are the synaptogenic factors released by astrocytes? Thrombospondins (TSPs) were recently identified as one of the key astrocyte-derived signals that promote CNS synaptogenesis [20]. TSPs, a family of large oligomeric extracellular matrix proteins, are secreted by cultured astrocytes and are normally expressed in immature, but not adult, astrocytes in vivo. Two TSP isoforms, TSP1 and 2, mimicked the effect of ACM in promoting the number of structural synapses formed by cultured RGCs [20]. Furthermore, immunodepletion of TSP1 and 2 from ACM inhibited the ability of astrocytes to induce synapse formation. Consistent with their role in synaptogenesis, TSP1/TSP2 double KO mice have significantly fewer cortical synapses in vivo [20]. The authors proposed that expression of TSP 1 and 2 acts as a permissive switch that helps time synaptogenesis during a distinct window in postnatal development. The identity of the neuronal TSP receptor and the specific signaling mechanisms by which TSP induces synaptogenesis are important questions for future investigation. Interestingly, TSP-induced synapses are presynaptically active, but are postsynaptically silent [20]. Given that astrocyte feeding layers and ACM can each induce both pre- and postsynaptic activity, astrocytes must also secrete a second signal that induces the insertion of AMPA receptors (AMPARs) into postsynaptic sites and converts these immature silent synapses into functional synapses.

Are there additional astrocyte-derived synaptogenic signals? Astrocyte-secreted cholesterol bound to apolipoprotein $\mathrm{E}$ (ApoE) particles was reported to enhance presynaptic function in RGC cultures [21]. Cholesterol increased presynaptic activity by increasing the quantal content of evoked synaptic responses. As cholesterol is required for lipid raft signaling, synaptic vesicle formation and synaptic function [22, 23], Mauch et al. [21] proposed that astrocytes are a major source of cholesterol and shuttle cholesterol to neurons to promote synapse formation. An important future question is whether this 'cholesterol shuttle' exists between neurons and astrocytes in vivo. One caveat of these in vitro studies is that neuronal cholesterol levels are likely abnormally low in autaptic RGC cultures. In fact, it was later reported that in higher-density RGC cultures, where cholesterol levels may not be as limiting, cholesterol enhanced synaptic efficacy, but failed to significantly increase synapse number, and had no effect on postsynaptic function [20].

Astrocyte-derived factors also control synaptic function in postnatal hippocampal neurons. Beattie et al. [24] found that glial-derived $\mathrm{TNF} \alpha$, a proinflammatory cyto- 
kine, significantly increased synaptic strength at excitatory synapses in postnatal hippocampal neurons by increasing the number of surface GluR1 AMPARs, whereas blockade of TNF $\alpha$ signaling had opposite effects. ACM mimicked the effects of TNF $\alpha$ in increasing the surface expression of AMPARs, and this effect was blocked by agents that prevent release of TNF $\alpha$. Thus, once excitatory synapses form, astrocyte-derived TNF $\alpha$ appears to be important for preserving synaptic strength, which may have important implications in both developmental and adult synaptic plasticity. Indeed, Stellwagen and Malenka [25] recently showed that glial-derived TNFo mediates homeostatic synaptic scaling, a process that uniformly adjusts synaptic strength in response to electrical activity independent of LTP or LTD. Interestingly, TNF $\alpha$ failed to induce postsynaptic function at RGC synapses [6], suggesting that the functional effects of astrocyte signals may be context-dependent or vary with the type of associated neurons.

Astrocyte-derived signals can also regulate the formation of inhibitory synapses by distinct pre- and postsynaptic mechanisms. This was recently explored in embryonic rat hippocampal neurons that were grown in the presence of ACM [26]. ACM significantly increased the number of inhibitory presynaptic terminals, the frequency of miniature IPSCs, and the number and synaptic localization of $\mathrm{GABA}_{\mathrm{A}}$ receptor $\left(\mathrm{GABA}_{\mathrm{A}} \mathrm{R}\right)$ clusters [26]. The authors initially hypothesized that the effects of astrocytes on inhibitory synapses were mediated by the neurotrophin BDNF and its receptor tyrosine receptor kinase B (TrkB), which are known modulators of inhibitory synapses. However, it turned out that the ability of ACM to promote the formation of inhibitory synapses was independent of BDNF/TrkB signaling, as ACM collected from TrkB receptor or BDNF KO astrocytes promoted a similar increase in the number of inhibitory synapses and $\mathrm{GABA}_{\mathrm{A}}$ R clusters as wild-type ACM. Yet BDNF secreted by neurons (and not astrocytes) was required for postsynaptic $\mathrm{GABA}_{\mathrm{A}} \mathrm{R}$ clustering. Thus, astrocytes apparently release a signal that modulates TrkB signaling in neurons to upregulate postsynaptic $\mathrm{GABA}_{\mathrm{A}}$ clusters. These findings suggest astrocytes can also indirectly regulate synapse development by modulating the expression of neuronal signaling molecules, such as neurotrophins and Trks. Consistent with this idea, Blondel et al. [27] recently found that vasoactive intestinal polypeptide (VIP) promotes synapse development and function through activity-dependent neurotrophic factor (ADNF), a protein secreted by VIP-stimulated astroglia. Astrocyte-derived ADNF acts directly on hippocampal neurons to promote the formation of excitatory synapses via the secretion of neurotrophin 3 (NT-3).

Taken together, these in vitro studies suggest that glia participate in the development and modulation of excitatory and inhibitory synapses through bidirectional signaling with neurons via the release of several factors at different stages of synapse development.

\section{Contact-Dependent Astrocyte-Neuron Signaling Regulates Synapse Development and Structural Plasticity}

Astrocytes extend thousands of fine processes that contact tens of thousands of synapses [2]. Like neurons, perisynaptic astrocytes express an array of cell adhesion molecules and proteins that could activate signaling systems in pre- and postsynaptic neurons. So far we have focused on secreted synaptogenic factors, but recent research suggests an important role for contact-dependent signaling between neurons and astrocytes in developmental plasticity.

Could direct adhesive interactions between astrocytic processes and neurons also promote synapse formation? This question was recently addressed in embryonic hippocampal neurons that were cultured on 'micro islands' of extracellular matrix proteins and overlaid with embryonic astrocytes [28]. Contact-dependent signaling between neurons and astrocytes was investigated by timelapse imaging using a GFP construct that specifically labeled the astrocytic plasma membrane. Indeed, local contact with astrocytes significantly increased the number and activity of presynaptic release sites, the amplitude of autaptic EPSCs, and also the number of excitatory synapses that formed in regions distal to the sites of astrocytic contact [28].

What are the molecular mechanisms underlying this contact-dependent signaling? Hama et al. [28] found that local glia-neuron contact triggered the activation integrin receptors and the arachidonic acid cascade in neurons. Activation of neuronal integrin receptors by astrocyte contact led to rapid and robust focal activation of neuronal protein kinase $\mathrm{C}(\mathrm{PKC})$ that subsequently spread throughout the entire neuron and promoted synaptogenesis. Astrocyte contact induced immediate (minutes) and persistent (days) activation of PKC, as measured by the phosphorylation and nuclear translocation of the major PKC substrate, MARCKS (myristoylated alanine-rich C kinase substrate). Pharmacological inhibition of PKC blocked MARCKS phosphorylation and impaired astro- 
cyte-contact induced synaptogenesis. While the astrocytic ligand that activates $\beta$ integrin signaling in neurons has not been identified, the authors propose that this form of local contact-dependent signaling between astrocytes and neurons can lead to the global facilitation of synaptogenesis [28]. Given that PKC signaling and astrocyte contact have been shown to regulate synaptic remodeling and function [29-32], it will be crucial to determine whether this mechanism of contact-dependent astrocyte-neuron signaling occurs in vivo.

Astrocyte contact also modulates the development of inhibitory synapses. Liu et al. [33] showed that local contact between neurons and astrocytes significantly increased the amplitude and density of $\mathrm{GABA}_{\mathrm{A}}$ currents in developing hippocampal neurons. This contact-dependent increase on GABAergic synaptic activity was dependent on calcium signaling in astrocytes. In addition, astrocytes were shown to regulate the chloride ion gradient in cultured spinal cord neurons and convert GABAergic synapses from excitatory to inhibitory [34]. This finding is particularly interesting given the importance of local GABAergic inhibitory circuits in both activity-dependent wiring of developing neural circuits and the consolidation of critical period plasticity $[35,36]$.

\section{Astrocytes Control Spine Dynamics and Structural Plasticity}

One hallmark of developmental synaptic plasticity is a dramatic change in spine morphology and motility. Spines, small protrusions on dendrites that receive excitatory glutamatergic input, are exquisitely sensitive to neuronal activity and environmental stimuli. Classic serial EM studies have shown that the fine processes of astrocytes intimately enwrap postsynaptic dendritic spines (fig. 1b). EM reconstructions in the mature hippocampus found that approximately $60 \%$ of synapses are in direct contact with astrocytes at any given time [1]. Importantly, contact between a postsynaptic spine and presynaptic axon is not sufficient to stabilize a spine [37-39], suggesting that perisynaptic astrocytes could play a role in promoting the stabilization and maturation of dendritic spines.

Recent live imaging experiments indicate that astrocytes show striking morphological plasticity at the synapse. Time-lapse confocal imaging of dendritic spines and astrocytes in acute slices prepared from GFAP:EGFP transgenic mice revealed that astrocytes rapidly extend and retract filopodia-like processes within a time scale of minutes [40]. Interestingly, astrocytic motility was only observed at active synaptic terminals (as measured by the stryl dye, FM1-43), suggesting that astrocytes are able to rapidly sense and respond to synaptic activity. A recent study by Haber et al. [31] further characterized the dynamic interplay between astrocytes and dendritic spines in organotypic hippocampal slices and found that changes in astrocyte motility were often coordinated with changes in spine motility (fig. 2). Surprisingly, astrocytic processes were generally more motile than their associated spines. Long-term imaging of astrocyte-spine dynamics revealed that astrocytes continually repositioned their processes on the surfaces of postsynaptic spines, and the extent of this reorganization was correlated with spine size; larger (more stable) spines had more stable contacts with astrocytic processes. Together, these findings suggest that structural modifications in astrocytes could regulate the positioning and access of signaling molecules to actively regulate synaptic structure and function.

One mechanism by which astrocytes could regulate dendritic spine dynamics is through Ephrin/EphA signaling. Hippocampal astrocytes and their processes express the ligand Ephrin-A3, while the EphA4 receptor tyrosine kinase is enriched on dendritic spines of pyramidal neurons (fig. 2c). Murai et al. [41] found that repulsive bidirectional Ephrin/Eph signaling between neurons and astrocytes regulates the morphology of postsynaptic spines. Activation of EphA4 by treatment with EphrinA3 fusion protein resulted in a significant reduction in spine length and a retraction of dendritic spines, whereas inhibiting EphA4-Ephrin interactions resulted in an increase in spine length and a distortion of spine shape. Consistent with these findings, irregularities in spine morphology were observed in hippocampal dominant negative EphA4 transfected neurons, or cultures prepared from EphA4 KOs [41]. The authors concluded that repulsive interactions between the Ephrin-A3 ligand on astrocytic processes and the EphA4 receptor on postsynaptic spines regulate spine morphology and plasticity. As EphA4 is normally developmentally downregulated in the brain $[42,43]$, it is tempting to speculate that inhibitory Ephrin-mediated neuron-glia synaptic signaling could play a role in the pruning of developing neural circuits.

Highly motile dendritic filopodia are frequently observed during postnatal development, whereas more stable dendritic spines are generally more prominent in the adult brain $[44,45]$. Time-lapse imaging studies from a number of laboratories support a 'filopodial' model of 

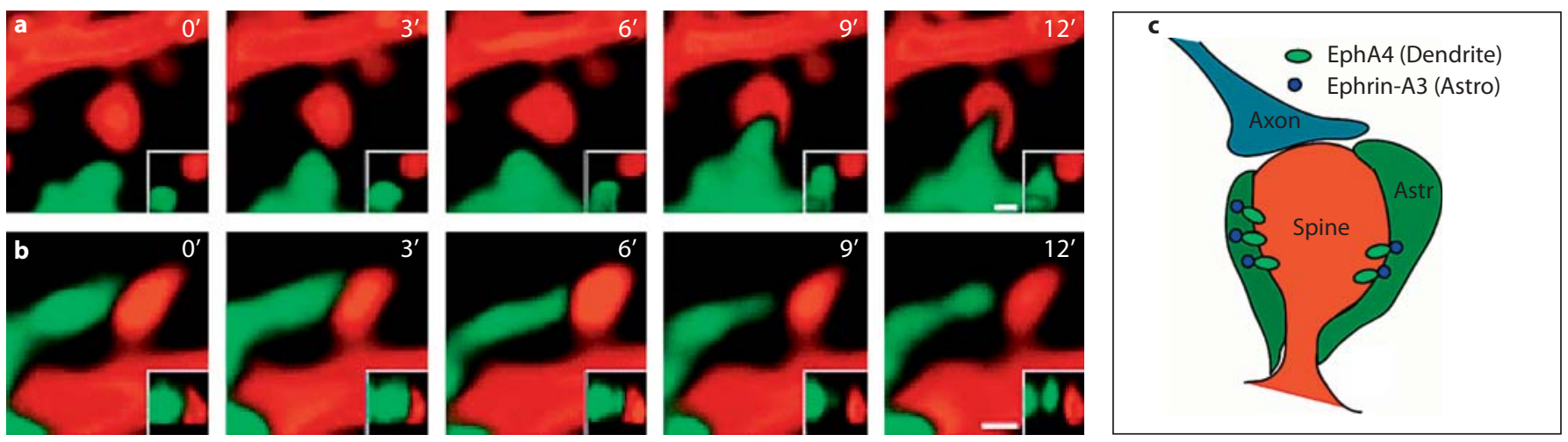

Fig. 2. Neuron-astrocyte interactions regulate spine morphology and plasticity. Dual time-lapse imaging of perisynaptic astrocytes (green) and postsynaptic dendritic spines (red) revealed dynamic structural interplay in situ. a An example of an astrocytic process extending toward and contacting a postsynaptic dendritic spine. b An example of rapid retraction of an astrocytic process from a

spine. Adapted from [31]. c One mechanism by which astrocytes regulate dendritic spine dynamics is through Ephrin/EphA signaling. Astrocytes and their processes express the ligand EphrinA3, while the EphA4 receptor tyrosine kinase is enriched on dendritic spines of pyramidal neurons $[32,41]$.

synaptogenesis, in which a subset of these motile dendritic protrusions are stabilized and converted to spines in the postnatal brain [46-48]. Could astrocytes regulate the development of immature dendritic filopodia to stable postsynaptic spines? Two-photon time-lapse imaging of astrocytic and dendritic processes revealed that dendritic protrusions that were in contact with astrocytic processes were morphologically more mature and generally more stable [32]. Both the motility of astrocytic processes and the Ephrin/EphA4 signaling between spines and astrocytes appear to be necessary for astrocyte-dependent stabilization of newly generated dendritic spines. Blocking astrocyte motility by the chronic perturbation of Racl-dependent signaling resulted in a decreased maturation of dendritic protrusions. Interestingly, inhibition of Ephrin/Eph neuron-astrocyte signaling destabilized new spines. This work led to a model in which astrocyte motility is a key regulator of the stabilization and maturation of filopodia-like protrusions into spines.

What are the functional consequences of astrocyte-mediated structural plasticity? This is still an open question in the developing brain, but several recent studies in the adult hypothalamo-neurohypophysial system provide new insight into this question. Astrocytes rapidly retract their processes that envelope somatodendritic synapses in responses to physiological and hormonal stimulation, such as lactation $[49,50]$. The withdrawal of astrocytic processes leads to an increase in the local glutamate concentration, which alters synaptic excitability, consistent with the role of astrocytes in clearing glutamate from the

synaptic cleft [50, 51]. Given that astrocytes release ATP, $\mathrm{D}$-serine and an array of gliotransmitters, this form of structural plasticity could have profound local effects on synaptic plasticity and function both in the developing and adult brain [for review, see 50]. Interestingly, astrocyte withdrawal resulted in a significant increase in the numbers of excitatory and inhibitory synapses [52], suggesting the interesting possibility that neurons and astrocytes might somehow compete for postsynaptic 'turf' to control the number of synaptic inputs a cell receives.

Taken all together, these studies suggest that contactmediated signaling between astrocytes and neurons is important for the structure and maintenance of synaptic connections and suggest a model in which physical and molecular interactions between neurons and perisynaptic astrocytes provide instructive cues that control synapse formation, morphology and plasticity.

\section{Role of Glia in Synapse Elimination and Refinement of Neural Circuits}

Synaptic pruning is essential for the precise wiring of the developing brain. Excess numbers of synapses are first generated to establish the initial wiring pattern, but the formation of mature, functional neural circuits requires the selective elimination and pruning of inappropriate synapses and the strengthening of appropriate synaptic connections. It is widely accepted that neural activity plays a crucial role in synaptic refinement, but 
surprisingly the specific molecular mechanisms that drive synapse elimination in the brain remain a mystery. The appearance of astrocytes in the postnatal brain coincides with this critical period of synapse remodeling, suggesting that in addition to providing synaptogenic signals, astrocytes might also provide instructive signals that could control which synapses are eliminated.

Recent research reveals that Bergmann glia (BG), specialized astrocytes that normally ensheath CF-Purkinje synapses, play a critical role in activity-dependent synapse elimination in the cerebellum. Purkinje neurons are initially innervated by multiple climbing fiber afferents, but by the second postnatal week the majority of these immature inputs are eliminated and only one CF input is strengthened [53]. This developmental shift from multito monoinnervation parallels synapse elimination at the mammalian neuromuscular junction [54]. Interestingly, BG have hundreds of subcellular compartments termed 'microdomains' that wrap around spines and respond to synaptic activity with increases in intracellular calcium [55].

BG express calcium-permeable AMPA-type glutamate receptors that lack a GluR2 subunit. When Iino et al. [56] molecularly converted these BG calcium-permeable receptors to calcium-impermeable AMPARs by in vivo transfection of the GluR2 gene, their processes retracted from CG-Purkinje synaptic spines. Disruption of BG calcium-permeable AMPARs also impaired the removal of synaptically released glutamate and prolonged the kinetics of glutamatergic synaptic transmission. Importantly, the normal developmental shift from multi- to monoinnervation was inhibited when astrocyte ensheathment was disrupted [56]. These results implicate glial calciumpermeable AMPARs are necessary for structural and functional interactions between BG and glutamatergic synapses that mediate developmental synapse elimination in vivo. These findings are consistent with in vitro studies that found that Purkinje neurons that were devoid of astrocyte ensheathment remain multi-innervated and display simple spike activity characteristics of immature neurons $[57,58]$. While the specific molecular mechanisms by which BG AMPARs mediate synapse elimination are not yet clear, these findings show a direct role for glial-neuronal signaling in developmental CNS synapse elimination, and highlight the developing cerebellum as an excellent model system to tease out the molecules involved.

Do astrocyes regulate developmental synaptic pruning in other areas of the CNS? A recent study by Stevens et al. [59] identified an unexpected and novel role for astro- cytes and proteins of the innate immune system in synapse elimination in the developing visual system. They found that a signal released by immature astrocytes induced the neuronal expression of $\mathrm{Clq}$, the initiating protein of the classical complement cascade. Using gene profiling, they found that mRNA for all $3 \mathrm{Clq}$ chains was significantly upregulated by astrocytes in purified RGC neurons. By RT-PCR and in situ hybridization, they found that C1q mRNA levels were highest in postnatal RGCs between P5 and P10, and declined significantly by P30. Importantly, immunohistochemistry revealed that $\mathrm{Clq}$ is highly expressed in the immature brain and retina, where it is localized to developing CNS synapses during the period of synapse elimination.

Clq's best known role in the innate immune system is to opsonize or 'tag' unwanted cells or debris for elimination. Could C1q similarly tag unwanted synapses for elimination in the developing brain? To investigate this possibility, Stevens et al. [59] focused on the development of retinogeniculate synapses, a well-established model system of synapse refinement and elimination [60-62]. Using a combination of neuroanatomical and electrophysiological techniques, they found that mice deficient in complement protein Clq or the downstream complement cascade protein $\mathrm{C} 3$ exhibit large sustained defects in CNS synapse elimination, as shown by the failure of anatomical refinement and eye-specific segregation of retinogeniculate connections in the dLGN. Importantly, electrophysiological recordings from LGN slices confirm this phenotype. Initially, dLGN neurons are innervated by multiple RGC afferents (up to 10) with weak synaptic strength, but by P30 each dLGN neuron normally receives strong, stable inputs from only 1-2 RGC axons [61]. In contrast, relay neurons in the dLGN in Clq- and C3deficient mice remain multi-innervated, even 2 weeks after eye opening [59]. Thus, classical complement proteins localize to CNS synapses during a discrete window of postnatal development and are required for synapse elimination in the developing retinogeniculate pathway (fig. 3). These findings add to the growing body of evidence that components of the immune system contribute to brain development and function [63].

How are complement 'tagged' synapses eliminated? One intriguing possibility is that they are eliminated by resident microglia. Activated microglia are the primary phagocytic cells in the brain and express high levels of the $\mathrm{C} 3$ complement receptor (also known as $\alpha \mathrm{M} \beta 2$ integrin, $\mathrm{cd} 11 \mathrm{~b} / \mathrm{cd} 18$, or mac1) [64]. Binding of $\mathrm{C} 3 \mathrm{~b}$ to $\mathrm{C} 3$ receptor is well known to induce phagocytosis. Microglia are also activated and recruited to synapses of motor neurons after 


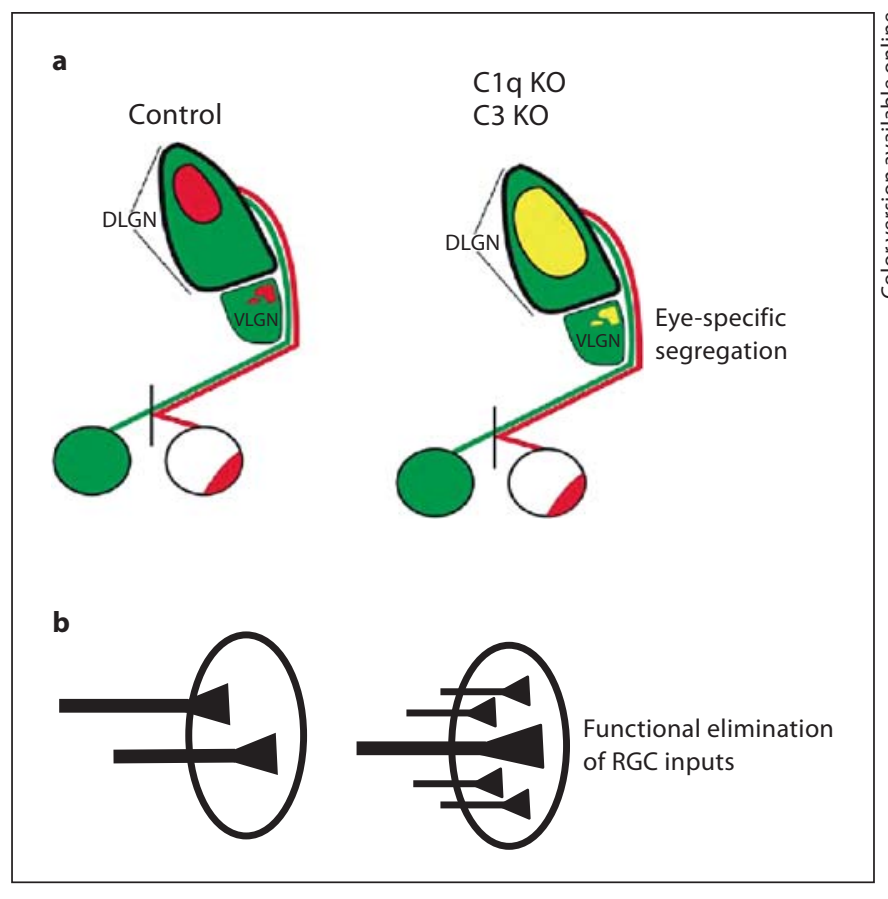

Fig. 3. Complement-dependent synapse elimination in the developing visual system. C1q, the initiating protein in the classical complement cascade, is expressed by postnatal neurons in response to immature astrocytes and is localized to developing CNS synapses. $\mathrm{Clq}$ and downstream complement protein $\mathrm{C} 3$ are required for both coarse-scale eye-specific segregation (a) and finescale functional elimination of retinogeniculate connections in the developing dLGN (b) [59].

axotomy in a process known as 'synaptic stripping', in which microglia rapidly engulf synapses terminating on these neurons [65]. It is not yet known if synaptic stripping is mediated by the complement cascade, or whether microglia actively phagocytose synapses during normal development. In the peripheral nervous system, synapse elimination at the NMJ is carried out by perisynaptic Schwann cells, which engulf and eliminate presynaptic terminals by a process known as 'axosome' shedding [66]. This process is not likely mediated by the complement cascade, as Schwann cells do not appear to express C3 complement receptors, and there was no obvious synapse elimination phenotype at the $\mathrm{NMJ}$ in complement $\mathrm{KO}$ mice [59].

These new findings suggest that a signal produced by immature astrocytes in the developing CNS induces the expression of complement protein C1q, thereby promoting the process of synaptic pruning. How complement is targeted to synapses and whether the complement cascade is regulated by neural activity are important questions for future investigation.

Neuron-Astrocyte Signaling during Developmental Plasticity

\section{Implications of Neuron-Astrocyte Signaling in CNS Injury and Neurodegenerative Disease}

Understanding the signals that help sculpt developing neural circuits could lend important insight into how to promote brain rewiring after injury or disease [67]. A unifying theme that has emerged from the research reviewed here is that immature astrocytes are special in that they can promote developmental plasticity of synaptic circuits via a variety of secreted and contact-dependent signals. Reactive astrocytes are thought to antigenically resemble immature astrocytes, suggesting that in the damaged brain, astrocytes could re-express many of the same signals that promote the formation and elimination of synapses during development [59] (fig. 4). Astrocytes (and microglia) become reactive following CNS injury and in neurodegenerative disease and can secrete an array of cytokines and inflammatory signals that can be harmful to neurons [68]. On the other hand, reactive glia can also play a protective role by releasing trophic factors, ECM molecules and signals that promote the clearance of toxic proteins like $A \beta$, and by phagocytosing degenerating axons and synapses [69]. Thus, reactive glia can both positively and negatively regulate synaptic function in the damaged and diseased brain.

Synapse elimination is necessary for the formation of mature functional neural circuits, but what if the same mechanisms that normally eliminate inappropriate synapses during development are recapitulated to promote destructive synapse loss in the adult brain? A common feature of neurodegenerative diseases is early synapse loss or dysfunction. For example, Alzheimer's disease (AD) is caused by a profound synapse degeneration, and growing evidence suggests that synapse loss can occur long before neuropathology and clinical symptoms in AD brains. In addition, complement expression and activation are known to be significantly upregulated following brain injury and in various neurodegenerative diseases, such as $\mathrm{AD}[70]$.

Could synapse loss at early stages of neurodegenerative disease involve a recapitulation of developmental synapse elimination mediated by the complement cascade? To address this question, Stevens et al. [59] used a mouse model of glaucoma to look at the timing and localization of $\mathrm{Clq}$ in the RGC and synaptic layers of retinas at various stages of disease (pre-glaucoma, early, moderate and late stage). They found that Clq is localized to synapses in the synaptic inner plexiform layer before significant synapse loss and RGC death, which supports the notion that complement is mediating synapse loss in early stages of dis-

Neurosignals 2008;16:278-288 
Fig. 4. Proposed model for the role of astrocytes in synaptic plasticity in the developing and damaged CNS. During development, immature astrocytes can promote synapse formation, elimination and structural plasticity of synaptic circuits via a variety of secreted and contact-dependent signals (left panel). These plasticity signals are downregulated in adult brain after the appropriate synaptic connections develop into stable, mature synaptic connections (middle panel). In the damaged or diseased brain, reactive astrocytes (and possibly microglia) re-express many of the same 'plasticity' signals that normally promote synapse formation and elimination during development, promoting synaptogenesis and synaptic pruning in the adult CNS.

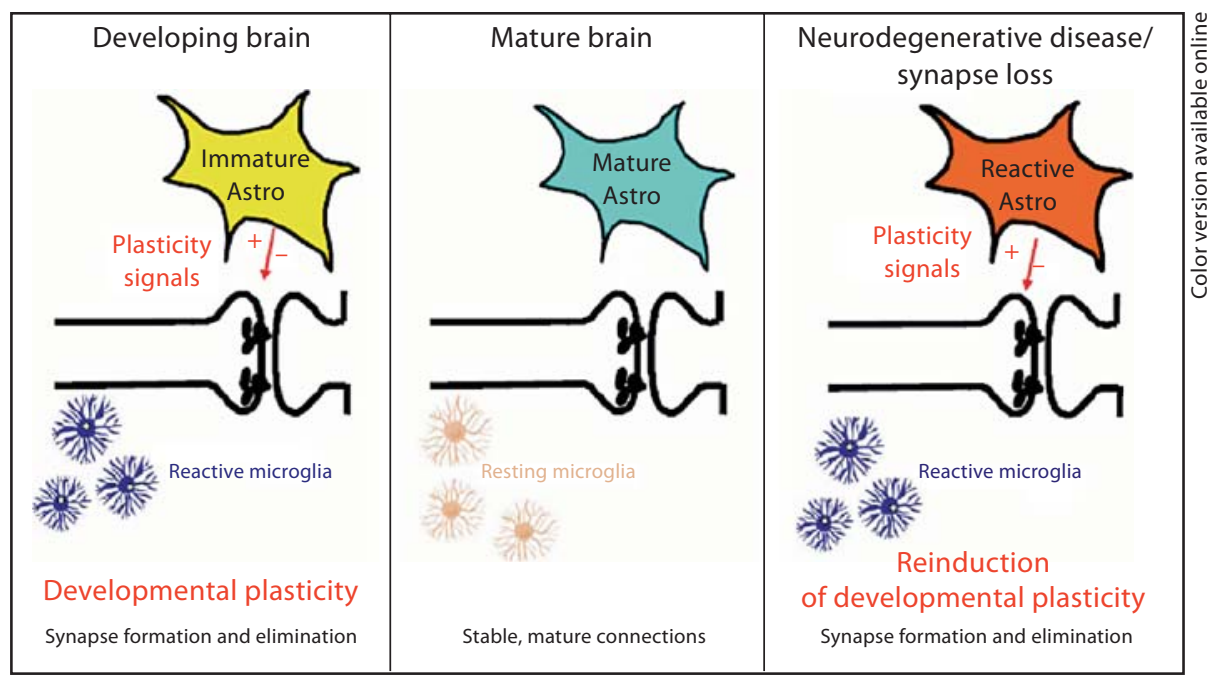

ease. These findings suggest that reactive astrocytes could re-express the signals that induce C1q expression in developing neurons. Identification of the signal(s) that triggers C1q upregulation in neurons could have therapeutic implications in diseases such as $\mathrm{AD}$ and glaucoma, where synapse loss occurs well before the onset of clinical symptoms.

Could reactive astrocytes also provide instructive signals that promote brain rewiring after CNS insult or disease? Increasing evidence suggests that adult brain may be more plastic than once thought. Recent research suggests the process of remapping and reconnection within the adult brain after injury, such as stroke, is an activitydependent process that involves the reactivation of developmental programs, such as synaptogenesis [71, 72]. Muller and Best's [12] finding that immature astrocytes reopened the window of critical period plasticity suggests that astrocytic signals could help rewire neural circuits in the less plastic, adult brain. Indeed, recent evidence suggests that astrocytes provide instructive signals that not only promote the differentiation of neural stem cells into neurons in the adult hippocampus, but also instruct these newly differentiated neurons to form new synaptic connections [73].

Insight into the molecular mechanisms by which astrocytes promote synaptogenesis during development could lead to therapeutic strategies to induce new synapses to form in the adult CNS after injury or disease. For example, TSP1 and 2, which are normally downregulated by mature astrocytes in vivo, are upregulated in reactive astrocytes [74]. Christopherson et al. [20] proposed that TSP1/2 may act as a permissive switch to time the win- dow of developmental synapse formation, suggesting that TSP $1 / 2$ could reopen the critical period window or perhaps even restore the capability to form new synapses in the adult brain. Interestingly, TSP4 is specifically expressed in mature astrocytes, suggesting that this mature isoform might play a role in synapse formation and plasticity in the adult brain.

\section{Conclusions}

It is becoming increasingly clear that astrocytes play a crucial and dynamic role at developing synapses. Astrocyte signals help promote both the formation and elimination of synapses as the brain wires up during development. In addition, dynamic physical and molecular interactions between astrocytes and neurons control the morphology and structural plasticity of dendritic spines. Once the appropriate synaptic connections are formed, astrocytes then modulate their function in the mature brain. Understanding the signals that help form and sculpt developing neural circuits could lend important insight into how to promote brain rewiring after injury or disease.

As astrocytes are essential for the normal structure and function of synapses, it has been difficult to dissect out their normal role in synapse development and plasticity in vivo. Recent advances in imaging techniques have shed new light on the dynamic interplay between neurons and astrocytes, but there is still a need for better molecular tools to study and manipulate key astrocytic molecules at the right places and times in vivo. Future studies may lead not only to a greater understanding of the role 
of astrocytes in regulating synaptic development and plasticity, but also to the development of novel potential ways to ameliorate the effects of CNS injuries or neurodegenerative diseases.

\section{Acknowledgements}

I would like to thank Ben A. Barres and the Larry L. Hillblom Foundation for their generous support of this work.

\section{References}

1 Ventura R, Harris KM: Three-dimensional relationships between hippocampal synapses and astrocytes. J Neurosci 1999;19:68976906.

$\checkmark 2$ Bushong EA, Martone ME, Jones YZ, Ellisman MH: Protoplasmic astrocytes in CA1 stratum radiatum occupy separate anatomical domains. J Neurosci 2002;22:183-192.

3 Haydon PG: GLIA: listening and talking to the synapse. Nat Rev Neurosci 2001;2:185193.

$\checkmark 4$ Volterra A, Meldolesi J: Astrocytes, from brain glue to communication elements: the revolution continues. Nat Rev Neurosci 2005;6:626-640.

5 Fields RD, Stevens-Graham B: New insights into neuron-glia communication. Science 2002;298:556-562.

-6 Ullian EM, Christopherson KS, Barres BA: Role for glia in synaptogenesis. Glia 2004;47: 209-216.

7 Allen NJ, Barres BA: Signaling between glia and neurons: focus on synaptic plasticity. Curr Opin Neurobiol 2005; 15:542-548.

-8 Nimmerjahn A, Kirchhoff F, Kerr JN, Helmchen F: Sulforhodamine 101 as a specific marker of astroglia in the neocortex in vivo. Nat Methods 2004;1:31-37.

-9 Benediktsson AM, Schachtele SJ, Green SH, Dailey ME: Ballistic labeling and dynamic imaging of astrocytes in organotypic hippocampal slice cultures. J Neurosci Methods 2005;141:41-53.

10 Halassa MM, Fellin T, Takano H, Dong JH, Haydon PG: Synaptic islands defined by the territory of a single astrocyte. J Neurosci 2007;27:6473-6477.

11 Fiacco TA, McCarthy KD: Astrocyte calcium elevations: properties, propagation, and effects on brain signaling. Glia 2006;54:676690.

12 Muller CM, Best J: Ocular dominance plasticity in adult cat visual cortex after transplantation of cultured astrocytes. Nature 1989;342:427-430.

13 Muller CM: A role for glial cells in activitydependent central nervous plasticity? Review and hypothesis. Int Rev Neurobiol 1992;34:215-281.

14 Pizzorusso T, Medini P, Berardi N, Chierzi S, Fawcett JW, Maffei L: Reactivation of ocular dominance plasticity in the adult visual cortex. Science 2002;298:1248-1251.
5 Ullian EM, Sapperstein SK, Christopherson KS, Barres BA: Control of synapse number by glia. Science 2001;291:657-661.

16 Barres BA, Silverstein BE, Corey DP, Chun LL: Immunological, morphological, and electrophysiological variation among retinal ganglion cells purified by panning. Neuron 1988;1:791-803.

17 Meyer-Franke A, Kaplan MR, Pfrieger FW, Barres BA: Characterization of the signaling interactions that promote the survival and growth of developing retinal ganglion cells in culture. Neuron 1995;15:805-819.

18 Pfrieger FW, Barres BA: Synaptic efficacy enhanced by glial cells in vitro. Science 1997; 277:1684-1687.

19 Ullian EM, Harris BT, Wu A, Chan JR, Barres BA: Schwann cells and astrocytes induce synapse formation by spinal motor neurons in culture. Mol Cell Neurosci 2004;25:241251.

20 Christopherson KS, Ullian EM, Stokes CC, Mullowney CE, Hell JW, Agah A, Lawler J, Mosher DF, Bornstein P, Barres BA: Thrombospondins are astrocyte-secreted proteins that promote CNS synaptogenesis. Cell 2005; 120:421-433.

21 Mauch DH, Nagler K, Schumacher S, Goritz C, Muller EC, Otto A, Pfrieger FW: CNS synaptogenesis promoted by glia-derived cholesterol. Science 2001;294:1354-1357.

22 Thiele C, Hannah MJ, Fahrenholz F, Huttner WB: Cholesterol binds to synaptophysin and is required for biogenesis of synaptic vesicles. Nat Cell Biol 2000;2:42-49.

23 Hering H, Lin CC, Sheng M: Lipid rafts in the maintenance of synapses, dendritic spines, and surface AMPA receptor stability. J Neurosci 2003;23:3262-3271.

24 Beattie EC, Stellwagen D, Morishita W, Bresnahan JC, Ha BK, Von Zastrow M, Beattie MS, Malenka RC: Control of synaptic strength by glial TNF $\alpha$. Science 2002;295: 2282-2285.

25 Stellwagen D, Malenka RC: Synaptic scaling mediated by glial TNF- $\alpha$. Nature $2006 ; 440$ : 1054-1059.

26 Elmariah SB, Oh EJ, Hughes EG, Balice-Gordon RJ: Astrocytes regulate inhibitory synapse formation via Trk-mediated modulation of postsynaptic $\mathrm{GABA}_{\mathrm{A}}$ receptors. J Neurosci 2005;25:3638-3650.
-27 Blondel O, Collin C, McCarran WJ, Zhu S, Zamostiano R, Gozes I, Brenneman DE, McKay RD: A glia-derived signal regulating neuronal differentiation. J Neurosci 2000; 20:8012-8020.

28 Hama H, Hara C, Yamaguchi K, Miyawaki A: PKC signaling mediates global enhancement of excitatory synaptogenesis in neurons triggered by local contact with astrocytes. Neuron 2004;41:405-415.

29 Daw MI, Chittajallu R, Bortolotto ZA, Dev KK, Duprat F, Henley JM, Collingridge GL, Isaac JT: PDZ proteins interacting with $\mathrm{C}$ terminal GluR2/3 are involved in a PKC-dependent regulation of AMPA receptors at hippocampal synapses. Neuron 2000;28: 873-886.

30 Fong DK, Rao A, Crump FT, Craig AM: Rapid synaptic remodeling by protein kinase $\mathrm{C}$ : reciprocal translocation of NMDA receptors and calcium/calmodulin-dependent kinase II. J Neurosci 2002;22:2153-2164.

31 Haber M, Zhou L, Murai KK: Cooperative astrocyte and dendritic spine dynamics at hippocampal excitatory synapses. J Neurosci 2006;26:8881-8891.

32 Nishida H, Okabe S: Direct astrocytic contacts regulate local maturation of dendritic spines. J Neurosci 2007;27:331-340.

- 33 Liu QY, Schaffner AE, Li YX, Dunlap V, Barker JL: Upregulation of $\mathrm{GABA}_{\mathrm{A}}$ current by astrocytes in cultured embryonic rat hippocampal neurons. J Neurosci 1996;16: 2912-2923.

-34 Li YX, Schaffner AE, Walton MK, Barker JL: Astrocytes regulate developmental changes in the chloride ion gradient of embryonic rat ventral spinal cord neurons in culture. J Physiol 1998;509:847-858.

35 Hensch TK: Critical period plasticity in local cortical circuits. Nat Rev Neurosci 2005;6: 877-888.

36 Ben-Ari Y: Excitatory actions of GABA during development: the nature of the nurture. Nat Rev Neurosci 2002;3:728-739.

37 Fischer M, Kaech S, Knutti D, Matus A: Rapid actin-based plasticity in dendritic spines. Neuron 1998;20:847-854.

38 Dunaevsky A, Blazeski R, Yuste R, Mason C: Spine motility with synaptic contact. Nat Neurosci 2001;4:685-686. 
-39 Lippman J, Dunaevsky A: Dendritic spine morphogenesis and plasticity. J Neurobiol 2005;64:47-57.

-40 Hirrlinger J, Hulsmann S, Kirchhoff F: Astroglial processes show spontaneous motility at active synaptic terminals in situ. Eur J Neurosci 2004;20:2235-2239.

-41 Murai KK, Nguyen LN, Irie F, Yamaguchi Y, Pasquale EB: Control of hippocampal dendritic spine morphology through ephrinA3/EphA4 signaling. Nat Neurosci 2003;6: 153-160.

42 Friedman GC, O’Leary DD: Eph receptor tyrosine kinases and their ligands in neural development. Curr Opin Neurobiol 1996;6: 127-133.

43 Tessier-Lavigne M: Eph receptor tyrosine kinases, axon repulsion, and the development of topographic maps. Cell 1995;82:345-348.

-44 Dunaevsky A, Tashiro A, Majewska A, Mason C, Yuste R: Developmental regulation of spine motility in the mammalian central nervous system. Proc Natl Acad Sci USA 1999;96:13438-13443.

45 Lendvai B, Stern EA, Chen B, Svoboda K: Experience-dependent plasticity of dendritic spines in the developing rat barrel cortex in vivo. Nature 2000;404:876-881.

46 Dailey ME, Smith SJ: The dynamics of dendritic structure in developing hippocampal slices. J Neurosci 1996;16:2983-2994.

-47 Ziv NE, Smith SJ: Evidence for a role of dendritic filopodia in synaptogenesis and spine formation. Neuron 1996;17:91-102.

-48 Portera-Cailliau C, Pan DT, Yuste R: Activity-regulated dynamic behavior of early dendritic protrusions: evidence for different types of dendritic filopodia. J Neurosci 2003; 23:7129-7142.

49 Hatton GI: Function-related plasticity in hypothalamus. Annu Rev Neurosci 1997;20: 375-397.

50 Bains JS, Oliet SH: Glia: they make your memories stick! Trends Neurosci 2007;30: 417-424.

- 51 Piet R, Poulain DA, Oliet SH: Modulation of synaptic transmission by astrocytes in the rat supraoptic nucleus. J Physiol Paris 2002; 96:231-236
2 Theodosis DT, Poulain DA: Maternity leads to morphological synaptic plasticity in the oxytocin system. Prog Brain Res 2001;133: 49-58.

53 Hashimoto K, Kano M: Functional differentiation of multiple climbing fiber inputs during synapse elimination in the developing cerebellum. Neuron 2003;38:785-796.

54 Sanes JR, Lichtman JW: Development of the vertebrate neuromuscular junction. Annu Rev Neurosci 1999;22:389-442.

55 Grosche J, Matyash V, Moller T, Verkhratsky A, Reichenbach A, Kettenmann H: Microdomains for neuron-glia interaction: parallel fiber signaling to Bergmann glial cells. Nat Neurosci 1999;2:139-143.

56 Iino M, Goto K, Kakegawa W, Okado H, Sudo M, Ishiuchi S, Miwa A, Takayasu Y, Saito I, Tsuzuki K, Ozawa S: Glia-synapse interaction through $\mathrm{Ca}^{2+}$-permeable AMPA receptors in Bergmann glia. Science 2001; 292:926-929.

57 Seil FJ: Interactions between cerebellar Purkinje cells and their associated astrocytes. Histol Histopathol 2001;16:955-968.

58 Drake-Baumann R, Seil FJ: Influence of functional glia on the electrophysiology of Purkinje cells in organotypic cerebellar cultures. Neuroscience 1999;88:507-519.

59 Stevens B, Allen NJ, Vazquez LE, Howell GR, Christopherson KS, Nouri N, Micheva KD, Mehalow A, Huberman AD, Stafford B, Sher A, Litke AM, Lambris JD, Smith SJ, John SWM, Barres BA: The classical complement cascade mediates CNS synapse elimination. Cell 2007;131:1164-1178.

60 Campbell G, Shatz CJ: Synapses formed by identified retinogeniculate axons during the segregation of eye input. J Neurosci 1992;12: 1847-1858.

61 Hooks BM, Chen C: Distinct roles for spontaneous and visual activity in remodeling of the retinogeniculate synapse. Neuron 2006; 52:281-291.

62 Ziburkus J, Guido W: Loss of binocular responses and reduced retinal convergence during the period of retinogeniculate axon segregation. J Neurophysiol 2006;96:27752784.

63 Boulanger LM, Shatz CJ: Immune signalling in neural development, synaptic plasticity and disease. Nat Rev Neurosci 2004;5:521531.
64 Gasque P, Singhrao SK, Neal JW, Wang P, Sayah S, Fontaine M, Morgan BP: The receptor for complement anaphylatoxin C3a is expressed by myeloid cells and non-myeloid cells in inflamed human central nervous system: analysis in multiple sclerosis and bacterial meningitis. J Immunol 1998; 160:35433554.

65 Schiefer J, Kampe K, Dodt HU, Zieglgansberger W, Kreutzberg GW: Microglial motility in the rat facial nucleus following peripheral axotomy. J Neurocytol 1999;28: 439-453.

66 Bishop DL, Misgeld T, Walsh MK, Gan WB, Lichtman JW: Axon branch removal at developing synapses by axosome shedding. Neuron 2004;44:651-661.

67 Wieloch T, Nikolich K: Mechanisms of neural plasticity following brain injury. Curr Opin Neurobiol 2006;16:258-264.

68 Davalos D, Grutzendler J, Yang G, Kim JV, Zuo Y, Jung S, Littman DR, Dustin ML, Gan WB: ATP mediates rapid microglial response to local brain injury in vivo. Nat Neurosci 2005;8:752-758.

69 Wyss-Coray T, Lin C, Yan F, Yu GQ, Rohde M, McConlogue L, Masliah E, Mucke L: TGF- $\beta_{1}$ promotes microglial amyloid- $\beta$ clearance and reduces plaque burden in transgenic mice. Nat Med 2001;7:612-618.

70 Tenner AJ, Fonseca MI: The double-edged flower: roles of complement protein $\mathrm{Clq}$ in neurodegenerative diseases. Adv Exp Med Biol 2006;586:153-176.

71 Carmichael ST: Cellular and molecular mechanisms of neural repair after stroke: making waves. Ann Neurol 2006;59:735742 .

72 Carmichael ST: Plasticity of cortical projections after stroke. Neuroscientist 2003;9:6475.

73 Song H, Stevens CF, Gage FH: Astroglia induce neurogenesis from adult neural stem cells. Nature 2002;417:39-44.

74 Lin TN, Kim GM, Chen JJ, Cheung WM, He YY, Hsu CY: Differential regulation of thrombospondin-1 and thrombospondin-2 after focal cerebral ischemia/reperfusion. Stroke 2003;34:177-186. 